\title{
Bijvoet pair's intensity ratio: Convergence of kinematic and dynamical diffraction
}

\author{
Xian-Rong Huang $\odot,{ }^{1, *}$ Lahsen Assoufid, ${ }^{1}$ Ru-Wen Peng, ${ }^{2, \dagger}$ Albert T. Macrander $\odot,{ }^{1}$ Thomas Gog, ${ }^{1}$ and Jungho Kim $\odot^{1}$ \\ ${ }^{1}$ Advanced Photon Source, Argonne National Laboratory, Argonne, Illinois 60439, USA \\ ${ }^{2}$ National Laboratory of Solid State Microstructures, School of Physics, and Collaborative Innovation Center of Advanced Microstructures, \\ Nanjing University, Nanjing 210093, China
}

(Received 1 March 2020; accepted 28 October 2020; published 1 December 2020)

\begin{abstract}
Anomalous diffraction of Bijvoet pairs (BPs) is a fundamental method of structure analyses in crystallography. We demonstrate that the diffraction intensity ratio of a BP always intrinsically equals the two structure factors' squared modulus ratio instead of the modulus ratio that has been believed to govern dynamical diffraction. High-resolution experiments of perfect quartz precisely proved this principle in pure dynamical diffraction. The underlying mechanism is that the elementary diffraction functions of BPs always obey the squared modulus ratio rule, which is universally valid for both kinematical and dynamical diffraction in any diffraction geometry. This fundamental mechanism clarifies one of the long-lasting ambiguities in x-ray crystallography and paves the way for various applications of high-resolution diffraction of BPs, particularly for large high-quality crystals.
\end{abstract}

DOI: 10.1103/PhysRevMaterials.4.123401

The intensity of x-ray diffraction from crystals depends on the structure factor modulus, $|F(\mathbf{H})|$, where $\mathbf{H}$ is the Bragg reflection vector. For crystals with centrosymmetry, $|F(\mathbf{H})| \equiv$ $|F(-\mathbf{H})|$, where $-\mathbf{H}$ is the opposite reflection. Consequently, the diffraction intensities of $\mathbf{H}$ and $-\mathbf{H}$ are identical, which is Friedel's law. For crystals without centrosymmetry, anomalous scattering can lead to $|F(\mathbf{H})| \neq|F(-\mathbf{H})|$. Then Friedel's law is broken and the two opposite reflections may produce different intensities. Here $\mathbf{H}$ and $\mathbf{- H}$ are called a Friedel pair [Fig. 1(a)] or, more generally, a Bijvoet pair (BP) if symmetry-equivalent reflections are included [1]. Accurate measurements of BP intensities are of fundamental importance for solving phases in structure analyses [2,3], for determining the absolute structures of molecules (chirality, handedness, and polarity) [4-8], for verifying the theoretical dispersion corrections to atomic scattering factors [9], for resonance spectroscopy, for observation of domains and polarization switching in (multi)ferroics [6,10-12], and for many other applications.

However, it is well known that there are two distinct principles governing $\mathrm{X}$-ray diffraction [13]. One is the kinematical diffraction principle for small or highly imperfect crystals, where the integrated diffraction intensity $I(\mathbf{H})$ is proportional to $|F(\mathbf{H})|^{2}$. The other is dynamical diffraction for large perfect

\footnotetext{
*xiahuang@anl.gov

†rwpeng@nju.edu.cn
}

Published by the American Physical Society under the terms of the Creative Commons Attribution 4.0 International license. Further distribution of this work must maintain attribution to the author(s) and the published article's title, journal citation, and DOI. crystals, where $I(\mathbf{H})$ is only proportional to $|F(\mathbf{H})|$. Hence, it seems that the diffraction intensity ratio of a BP should be

$$
\frac{I(\mathbf{H})}{I(-\mathbf{H})}=\frac{|F(\mathbf{H})|^{2}}{|F(-\mathbf{H})|^{2}}
$$

for small or imperfect crystals and

$$
\frac{I(\mathbf{H})}{I(-\mathbf{H})}=\frac{|F(\mathbf{H})|}{|F(-\mathbf{H})|}
$$

for large perfect crystals. The difference between Eqs. (1) and (2) can be remarkable. If it were true, its influence could be felt in most sectors of crystallography. Although many phasing methods using BPs are for small biological crystals that are believed to be dominated by kinematical diffraction, in fact, there is no clear boundary between kinematical and dynamical diffraction. Particularly in the reflection geometry, $\mathrm{x}$-ray penetration can be only a few tens of micrometers due to extinction, and diffraction from such crystals can be a mixed kinematical and dynamical diffraction process.

Nevertheless, the classical dynamical theory has already indicated that Eq. (1) may be valid even in dynamical diffraction (e.g., [14-16]), but this conclusion was trivially derived or described in very complex or misleading formulas, which are further complicated by various scenarios (such as absorption and nonabsorption crystals, integrated or nonintegrated intensities, different diffraction geometries, etc.), resulting in much confusion in the literature. On the other hand, since the proportionality $I(\mathbf{H}) \propto|F(\mathbf{H})|$ in dynamical diffraction has been dominantly accepted, therefore, it is still widely believed that Eq. (2) is valid for large perfect crystals $[6,17,18]$, and that for relatively small crystals, $I(\mathbf{H}) / I(-\mathbf{H})$ could take some intermediate values between Eqs. (1) and (2) [19].

In this paper, we use both high-resolution experiments and simple but systematic formulas to demonstrate that $I(\mathbf{H}) / I(-\mathbf{H})$ strictly follows Eq. (1) in both dynamical and 


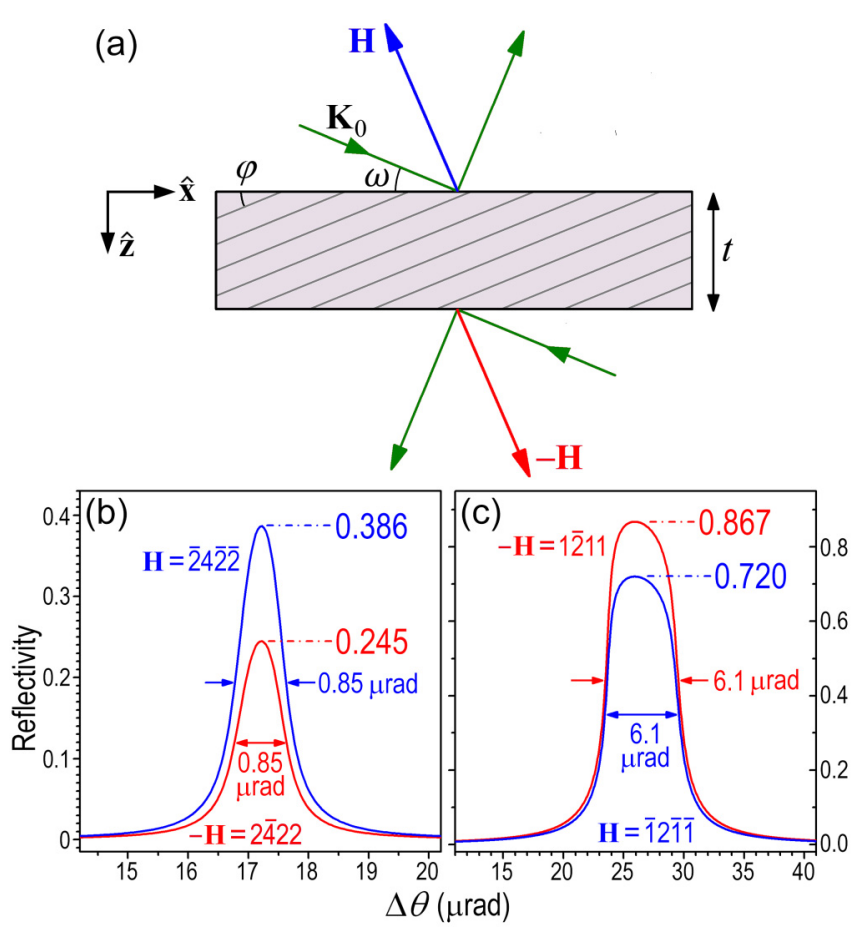

FIG. 1. (a) X-ray diffraction of BPs in the same geometry. $\varphi$ is the asymmetric angle ( $\varphi=0$ for symmetric reflection). $\theta=\omega+\varphi$. (b) Theoretical Darwin curves of the $\overline{2} 4 \overline{2} \overline{2} / 2 \overline{4} 22 \mathrm{BP}$ of right-handed quartz with the same Bragg angle $\theta_{B}=43.54^{\circ}$. Both the peak reflectivity ratio and the integrated intensity ratio are $0.386 / 0.245=$ 1.58. (c) $\overline{1} 2 \overline{1} \overline{1} / 1 \overline{2} 11 \mathrm{BP}$ with $\theta_{B}=20.15^{\circ}$. The intensity ratio is $0.720 / 0.867=0.83 . \varphi=0, \sigma$ polarization (for all simulations in this paper).

kinematical diffraction, thus clarifying one of the long-lasting ambiguities in X-ray crystallography. This is particularly important for anomalous diffraction of nearly perfect large crystals that can achieve much higher resolution and reliability than small crystals. The related techniques can thus be used to accurately study various fundamental physics and have many practical applications.

We study right-handed noncentrosymmetric $\alpha$ quartz, which belongs to the trigonal crystal system with space groups $P 3_{2} 21$ (no. 154). In the right-handed hexagonal coordinate system, the unit cell contains three $\mathrm{Si}$ atoms at $(u, 0,0)$, $(-u,-u, 1 / 3)$, and $(0, u, 2 / 3)$ and six $\mathrm{O}$ atoms at $(x, y, z)$, $(y, x, 2 / 3-z), \quad(-y, x-y, 2 / 3+z), \quad(-x, y-x, 1 / 3-z)$, $(y-x,-x, 1 / 3+z)$, and $(x-y,-y,-z)$, where $u=0.4699$, $x=0.4141, y=0.2681$, and $z=0.1188$. The lattice constants are $a=b=4.9134 \AA, c=5.4052 \AA, \alpha=\beta=90^{\circ}$, and $\gamma=120^{\circ}$. The Debye temperatures of the $\mathrm{Si}$ and $\mathrm{O}$ atoms are 517 and $476 \mathrm{~K}$, respectively [20,21]. The anomalous scattering factor $f_{\mathrm{Si}}^{\prime}+i f_{\mathrm{Si}}^{\prime \prime}$ of $\mathrm{Si}$ has appreciable values in the energy range $E \leqslant 14 \mathrm{keV}$ (while negligible for the $\mathrm{O}$ atoms). For instance, at $E=8.05 \mathrm{keV}, f_{\mathrm{Si}}^{\prime}+i f_{\mathrm{Si}}^{\prime \prime}=0.297+i 0.325$ (electrons), and it increases with decreasing $E$ (e.g., $f_{\mathrm{Si}}^{\prime}+$ $i f_{\mathrm{Si}}^{\prime \prime}=0.42+i 0.795$ at $\left.E=5 \mathrm{keV}\right) \cdot f_{\mathrm{Si}}^{\prime}+i f_{\mathrm{Si}}^{\prime \prime}$ can cause some BPs of quartz to have significantly different structure factor moduli.

For example, at $E=8.05 \mathrm{keV}$, the structure factors of reflection $\mathbf{H}=\overline{2} 4 \overline{2} \overline{2}$ and its opposite reflection
$-\mathbf{H}=2 \overline{4} 22$ are $F(\mathbf{H})=-0.454+i 1.615$ and $F(-\mathbf{H})=$ $-0.717-i 1.983$, respectively, with $|F(\mathbf{H}) / F(-\mathbf{H})|^{2}=1.58$ and $|F(\mathbf{H}) / F(-\mathbf{H})|=1.26$. For the base reflections, the two structure factors are $F(\mathbf{H}=\overline{1} 2 \overline{1} \overline{1})=6.94-i 7.47$ and $F(-\mathbf{H}=1 \overline{2} 11)=5.95+i 7.14$ with $|F(\mathbf{H}) / F(-\mathbf{H})|^{2}=$ 0.831 and $|F(\mathbf{H}) / F(-\mathbf{H})|=0.911$. Based on these structure factors, we have calculated the corresponding Darwin curves in Figs. 1(b) and 1(c). Surprisingly, the intensity ratios of the BPs exactly follow Eq. (1) instead of Eq. (2), though the calculations are rigorously based on the dynamical theory of perfect semi-infinite crystals $(t=\infty)$. Interestingly, the Darwin widths of the BP are always the same [0.86 and 6.1 $\mu \mathrm{rad}$ in Figs. 1(b) and 1(c), respectively], indicating that the two curves have the same shape. Indeed, if we scale the intensities of reflection $-\mathbf{H}$ by the factor $|F(\mathbf{H}) / F(-\mathbf{H})|^{2}$, the two curves become identical, which means that not only does the peak reflectivity ratio follow Eq. (1) but the intensity ratio at each angle $\Delta \theta$ also follows Eq. (1). Consequently, the integrated intensity ratio also satisfies Eq. (1).

In order to verify Eq. (1), we acquired a number of large synthetic quartz crystals $\left(220 \times 80 \times 40 \mathrm{~mm}^{3}\right)$ from Tokyo Denpa Co., Ltd [20], from which a series of quartz ( $\overline{1} 2 \overline{1} \overline{1})$ wafers $(\approx 4 \mathrm{~mm}$ thick) were cut and carefully lapped, etched, and polished. Afterwards, synchrotron white-beam x-ray topography characterization was performed on these wafers. It was found that most of the wafers are nearly perfect with no dislocations or other crystalline defects, as shown in Fig. 2(a). Such perfect wafers were used in the following experiments, which ensures that $\mathrm{x}$-ray diffraction from these wafers is purely dynamical diffraction without kinematical diffraction contributions.

The $\overline{2} 4 \overline{2} \overline{2} / 2 \overline{4} 22$ BP of the wafers was first studied by the double-crystal diffraction method with an asymmetric $\mathrm{Si}$ (333) crystal as the beam conditioner [Fig. 2(b)]. The X-ray beam was always set at $E=8.05 \mathrm{keV}$ [except Fig. 2(a)] by the upstream Si (111) double-crystal monochromator (DCM). The Bragg angle of the $\mathrm{Si} 333$ reflection is $47.47^{\circ}$, which is close to the Bragg angle of $43.54^{\circ}$ of quartz $\overline{2} 4 \overline{2} \overline{2}$ and $2 \overline{4} 22$ reflections. The asymmetric angle of the $\mathrm{Si}$ (333) crystal is $\varphi=45.47^{\circ}$ such that the incidence angle is $2^{\circ}$ and the vertical beam expansion factor is the asymmetric factor $|b|=28.6$. Afterwards, the x-ray footprint on the quartz crystal is limited to $15 \times 15 \mathrm{~mm}^{2}$. The unique advantage of double-crystal diffraction is that when the two reflections have similar Bragg angles the two diffraction bands in the DuMond diagram [inset of Fig. 2(b)] are nearly parallel to each other. Rocking the quartz sample is then equivalent to scanning the diffraction band of quartz across the Si band along the horizontal direction $(\theta)$, and obviously the scanning is nearly identical for any wavelength $(\lambda)$ around the Bragg conditions. Consequently, the convolution of the two crystals is almost independent of the divergence and spectrum of the incident beam. Hence, the rocking curve (RC) measured is nearly independent of the incidence conditions, and is thus very accurate.

The dotted lines in Figs. 2(c) and 2(d) are the measured double-crystal RCs of $\overline{2} 4 \overline{2} \overline{2}$ and $2 \overline{4} 22$, respectively. Under stable experimental conditions, the measurements were repeated many times with different quartz samples. For the same reflection, all the RCs have the same shape with the peak intensity variation less than $3 \%$. The fact that the $\overline{2} 4 \overline{2} \overline{2}$ 

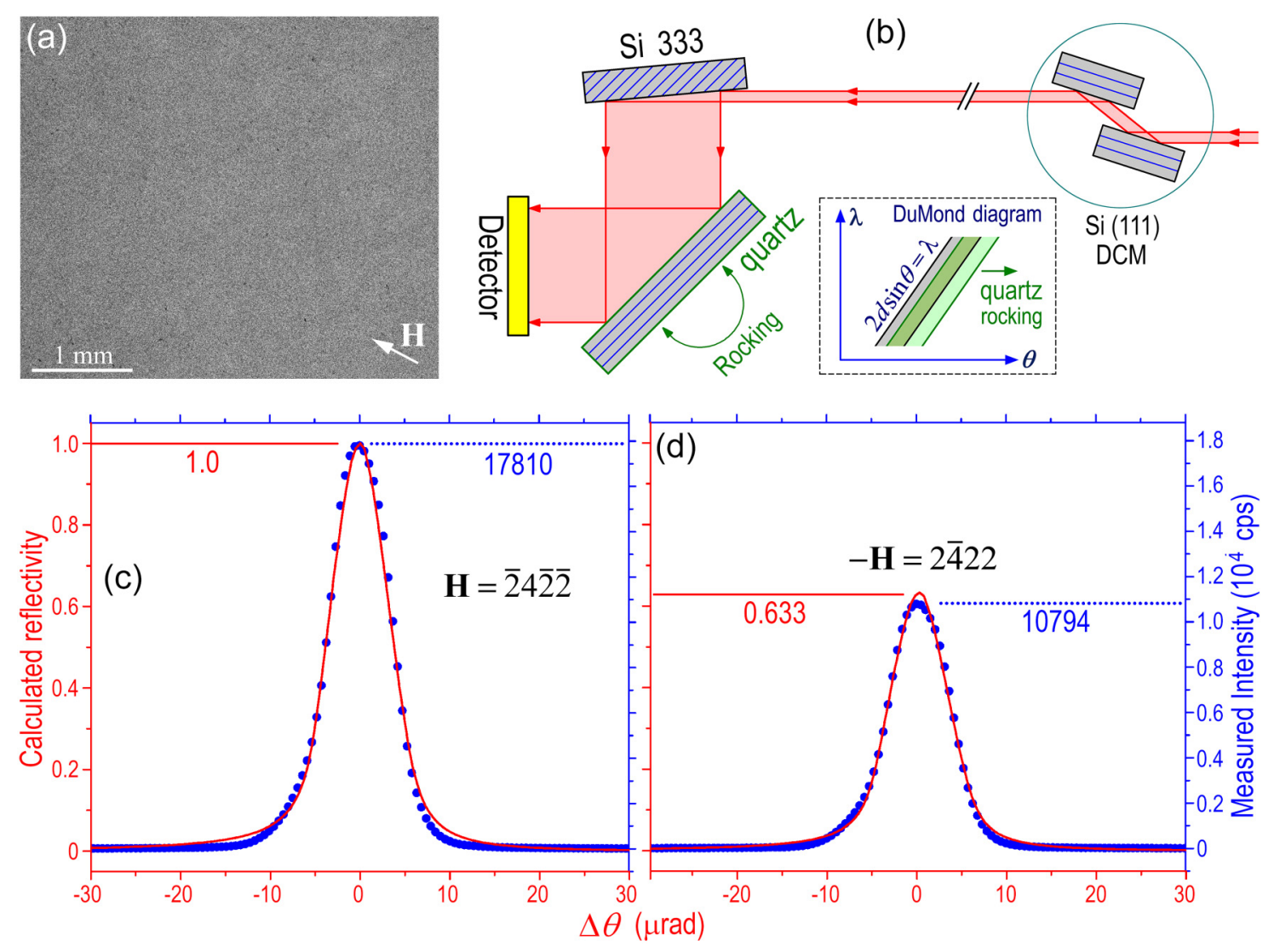

FIG. 2. (a) White-beam reflection topograph of one of the quartz ( $\overline{1} 2 \overline{1} \overline{1})$ wafers. The diffraction vector of this reflection is $\mathbf{H}=2 \overline{1} \overline{1} 0$ at energy $E=10.3 \mathrm{keV}$. (b) Schematic of double-crystal diffraction. (c) Measured and simulated RCs of $\overline{2} 4 \overline{2} \overline{2}$ symmetric reflection. The calculated peak reflectivity is normalized to unity. (d) Measured and simulated RCs of symmetric $2 \overline{4} 22$ reflection. The measured FWHMs in (c) and (d) are 7.86 and $7.95 \mu \mathrm{rad}$, while the calculated FWHM is $7.67 \mu \mathrm{rad}$. The units and scales of the reflectivity and intensity in (c) and (d) are identical.

reflection is stronger than $2 \overline{4} 22$ unambiguously reveals that the crystals are right-handed quartz. Left-handed quartz is the inverted structure of right-handed quartz [20], so reflection $\overline{2} 4 \overline{2} \overline{2}$ should be weaker than $2 \overline{4} 22$. This shows that anomalous diffraction from large perfect crystals can be conveniently used to accurately determine the handedness of chiral crystals (or polarity of ferroic crystals) without the need to select a wavelength close to the absorption edge due to the high accuracy, which is an important application that can be carried out even with laboratory x-ray sources.

The red lines in Figs. 2(c) and 2(d) are the simulated RCs based on the dynamical theory. The divergence of the beam incident on the Si (333) beam conditioner was set to $40 \mu \mathrm{rad}$ for the simulation. Here the fact that the RCs are much wider than the Darwin curves in Fig. 1(b) is due to the convolution with the Si 333 reflection that has a wider Darwin width and a wider bandwidth. Apparently, the measured RCs are very close to the simulated ones. Of most importance is that the measured peak intensity ratio is 1.65 , very close to the calculated ratio 1.58 . Since the two measured curves in Figs. 2(c) and 2(d) have the same shape, the ratio of the integrated intensity is also 1.65 . This verifies that the diffraction intensities of the quartz $\overline{2} 4 \overline{2} \overline{2} / 2 \overline{4} 22 \mathrm{BP}$ agree well with Eq. (1) under dynamical diffraction conditions. The relative deviation of the measured ratio from Eq. (1) is less than 5\% [while it deviates from the prediction of 1.28 from Eq. (2) by $31 \%$ ]. The high accuracy of the experiments is also indicated by the excellent agreement between the measured and calculated RC widths. In particular, the almost identical widths of the two measured RCs indicate that the crystalline qualities of the two opposite surfaces of the (i $2 \overline{1} \overline{1})$ wafers are the same, i.e., the intensity difference is indeed caused by the anomalous scattering instead of surface imperfections or defects (if any). Note that when the crystal is defective the defects or surface imperfections can remarkably change the rocking curves, which will make the measurements completely unreliable.

Next, we removed the Si (333) beam conditioner and performed the simple single-axis diffraction, as schematically shown in Fig. 3(a). The blue and red solid lines in Fig. 3(b) are the measured RCs of $\overline{2} 4 \overline{2} \overline{2}$ and $2 \overline{4} 22$, respectively, and the dashed lines are the corresponding dynamical-theory simulations that include convolution with the DCM. The single-axis diffraction process has lower accuracy and the rocking curve strongly depends on the incident beam conditions (e.g., the incidence divergence, spectrum, etc.). Compared to Figs. 2(c) and 2(d), therefore, here the measured full widths at half maximum (FWHMs) are much wider, $134 \mu \mathrm{rad}$, for both measured RCs. The deviation between the measurements and simulations is noticeable, as shown particularly by the elevated tails. However, the shapes of the two measured curves are the same, 
(a)
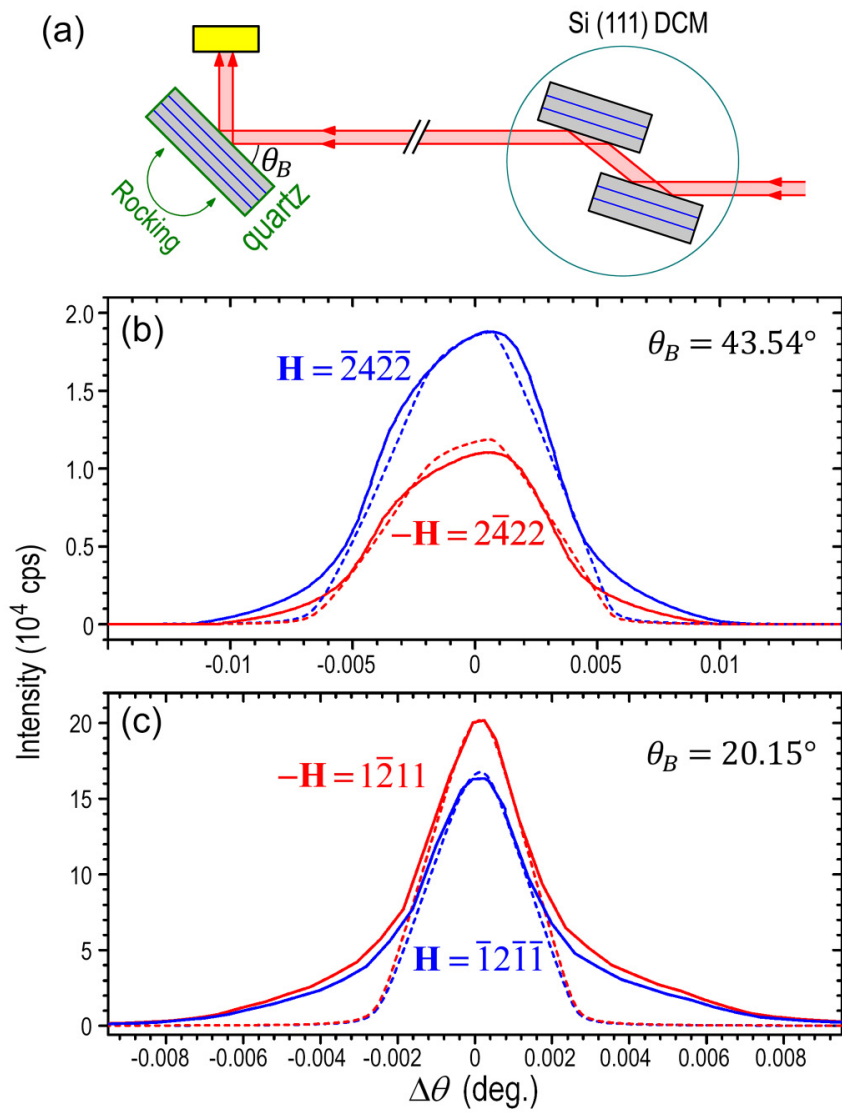

FIG. 3. (a) Schematic of single-axis diffraction. (b) Measured (solid line) and simulated (dashed line) RCs of symmetric reflections $\overline{2} 4 \overline{2} \overline{2}$ (blue) and $2 \overline{4} 22$ (red) by the single-axis diffraction setup in (a). (c) The corresponding RCs of symmetric $\overline{1} 2 \overline{1} \overline{1} / 1 \overline{2} 11$ reflections.

indicating that the deviation is purely caused by the incident beam conditions or beamline instruments, not by crystalline imperfections (if any) of the quartz samples. The measured intensity ratio of the two RCs is 1.70 , while the ratio between the two theoretical RCs is still 1.58. The relative difference between the two ratios is $7.6 \%$, which is still close enough to indicate that the single-axis diffraction intensity ratio also agrees with Eq. (1) instead of Eq. (2) because the deviation from the latter is $35 \%$. Therefore, Figs. 3(b), 2(c), and 2(d) show that, although the conditions of the incident beam and the instruments can remarkably change the RCs, the intensity ratio remains the same as predicted by Eq. (1), indicating that this is the intrinsic property of the BP.

The solid lines in Fig. 3(c) are the RCs of the base reflections $\overline{1} 2 \overline{1} \overline{1} / 1 \overline{2} 11$ of the ( $\overline{1} 2 \overline{1} \overline{1})$ wafers measured with the setup in Fig. 3(a), and the dashed lines are the simulations. Here the Bragg angle $\theta_{B}$ of quartz is smaller but closer to the Bragg angle $\left(14.22^{\circ}\right)$ of the DCM. Thus, the RC widths $(\approx 45 \mu \mathrm{rad})$ are smaller than those in Fig. 3(b). Although in Fig. 3(c) the tails of the measured Bragg peaks are again much higher than the simulations, the two curves still have the same shape, indicating that the tails are again caused by the beamline instruments. Equation (1) and Fig. 1(c) have predicted that the $\overline{1} 2 \overline{1} \overline{1}$ reflection from the front surface of the $(\overline{1} 2 \overline{1} \overline{1})$ wafer is weaker than the reflection $1 \overline{2} 11$ from the back surface, which is opposite to the situation of the above second-order reflection pair $\overline{2} 4 \overline{2} \overline{2} / 2 \overline{4} 22$. Figure 3(c) correctly reveals this inequality, where the measured intensity ratio of the $\overline{1} 2 \overline{1} \overline{1} / 1 \overline{2} 11$ pair is 0.81 , very close to the theoretical value of 0.83 predicted by Eq. (1).

Therefore, Figs. 1-3 clearly demonstrate that the dynamical-diffraction intensity ratio between a BP is always governed by Eq. (1) under different diffraction conditions. The underlying mechanism can be understood from the following plane-wave dynamical theory.

In Fig. 1(a), the incident wave vector $\mathbf{K}_{0}$ is in the $x z$ plane. Near the Bragg condition of reflection $\mathbf{H}$, the incident plane wave activates two wave fields in the crystal, $\quad \mathbf{D}_{0}^{(1,2)} \exp \left[-i\left(\mathbf{K}_{0}-K \delta_{1,2} \hat{\mathbf{z}}\right) \cdot \mathbf{r}\right]+$ $\mathbf{D}_{H}^{(1,2)} \exp \left[-i\left(\mathbf{K}_{0}+\mathbf{H}-K \delta_{1,2} \hat{\mathbf{z}}\right) \cdot \mathbf{r}\right], \quad$ where $\quad K=2 \pi / \lambda$. Define a parameter representing the deviation of the incidence angle $\omega$ and the wavelength $\lambda$ from the Bragg conditions:

$$
\begin{aligned}
\eta= & \frac{1}{C \Gamma[F(\mathbf{H}) F(-\mathbf{H})|b|]^{1 / 2}}\left\{\frac{\lambda}{d}\left[\sin (\omega+\varphi)-\frac{\lambda}{2 d}\right]\right. \\
& \left.-\frac{\Gamma \mathrm{F}(0)(1+|b|)}{2}\right\},
\end{aligned}
$$

where $|b|=\sin \left(\theta_{B}+\varphi\right) / \sin \left(\theta_{B}-\varphi\right)$ is the asymmetric factor, $d$ is the lattice spacing, $C$ is the polarization factor [equal to 1 and $\cos \left(2 \theta_{B}\right)$ for $\sigma$ and $\pi$ polarization, respectively], and $\Gamma=r_{e} \lambda^{2} /(\pi \mathrm{V})\left(r_{e}\right.$ is the classical electron radius and $V$ is the unit-cell volume). Compared with the conventional definition of $\eta[15,17]$, here Eq. (3) has the advantages that it is a two-dimensional function of both $\omega$ and $\lambda$ and is valid even when $\theta_{B} \rightarrow 90^{\circ}$ [22]. Then one can derive

$$
\begin{aligned}
\delta_{1,2}= & \frac{C \Gamma[F(\mathbf{H}) F(-\mathbf{H})]^{1 / 2}}{2 \sin \left(\theta_{B}-\varphi\right)|b|^{1 / 2}}\left[\eta \pm\left(\eta^{2}-1\right)^{1 / 2}\right] \\
& +\frac{\Gamma F(0)}{2 \sin \left(\theta_{B}-\varphi\right)},
\end{aligned}
$$

where the imaginary parts of $\delta_{1}$ and $\delta_{2}$ always have opposite signs. The ratios of the internal wave amplitudes are

$$
r_{1,2}=D_{H}^{(1,2)} / D_{0}^{(1,2)}=\frac{\Psi_{1,2}(\eta)}{|b|^{1 / 2}}\left[\frac{F(\mathbf{H})}{F(-\mathbf{H})}\right]^{1 / 2},
$$

where

$$
\Psi_{1,2}(\eta)=-\left[\eta \pm\left(\eta^{2}-1\right)^{1 / 2}\right]
$$

Here we first let + and - of the \pm signs in Eqs. (4)-(6) correspond to wave field 1 and 2, respectively. However, if $\operatorname{Im}\left(\delta_{1}\right)<0$, we swap the indices of the two wave fields to ensure $\operatorname{Im}\left(\delta_{1}\right)>0$ and $\operatorname{Im}\left(\delta_{2}\right)<0$. For a semi-infinite crystal $(t \rightarrow \infty)$, only wave field 1 exists, and the Bragg reflectivity is

$$
R_{\infty}(\mathbf{H}, \omega, \lambda)=|b|\left|r_{1}\right|^{2}=\left|\Psi_{1}\right|^{2}\left|\frac{F(\mathbf{H})}{F(-\mathbf{H})}\right| .
$$

Note that Eqs. (4) and (6) are symmetric about $\mathbf{H}$ and $-\mathbf{H}$. Under identical geometry, therefore, the reflectivity of the opposite reflection $-\mathbf{H}$ is $R_{\infty}(-\mathbf{H}, \omega, \lambda)=\left|\Psi_{1}\right|^{2}|F(-\mathbf{H}) / F(\mathbf{H})|$. 
Consequently, it is rigorous that

$$
\frac{R_{\infty}(\mathbf{H}, \omega, \lambda)}{R_{\infty}(-\mathbf{H}, \omega, \lambda)}=\frac{|F(\mathbf{H})|^{2}}{|F(-\mathbf{H})|^{2}} .
$$

This is the elementary diffraction function showing that the reflectivity ratio of a $\mathrm{BP}$ is strictly equal to the ratio of the squared moduli of the two structure factors for a plane wave with any incidence direction $\omega$ and any wavelength $\lambda$ near the Bragg conditions. In experiments, the measured intensities are usually integrated or convoluted intensities. However, if the integration or convolution conditions of $\mathbf{H}$ and $\mathbf{- H}$ are the same, such intensities still strictly preserve the ratio $|F(\mathbf{H})|^{2} /|F(-\mathbf{H})|^{2}$ because they are simply integrations of the elementary functions. Therefore, for comparing diffraction of BPs, there is no need to distinguish between plane-wave, integrated, or convoluted intensity ratios since they have the same value. Thus, Eq. (8) proves Eq. (1) for pure dynamical diffraction of BPs from thick crystals. The elementary function Eq. (8) also explains why each RC pair in Figs. 1-3 has the same intensity ratio $|F(\mathbf{H})|^{2} /|F(-\mathbf{H})|^{2}$ at any angle $\Delta \theta$ such that each $\mathrm{RC}$ pair has the same shape and FWHM. This is a unique property of BPs, which is different from the general diffraction principles for comparing different reflections other than BPs, where only the integrated intensity is proportional to $|F(\mathbf{H})|^{2}$ (kinematical diffraction) or $|F(\mathbf{H})|$ (dynamical diffraction) while the elementary function does not necessarily have this proportionality.

For example, in the dynamical theory of nonabsorption crystals, the Darwin curve is a universal flat-plateau curve in terms of the normalized parameter $\eta: R_{\infty}=$ 1 for $|\eta| \leqslant 1$ and $R_{\infty}=\left[|\eta|-\left(\eta^{2}-1\right)^{1 / 2}\right]^{2}$ for $|\eta|>1$ $[15,17]$. Then the integrated intensity is $I(\mathbf{H})=\int R_{\infty} d \omega=$ $\sqrt{|b|}|C| \Gamma|F(\mathbf{H})| /\left(3 \sin 2 \theta_{B}\right)$. This is the origin of $I(\boldsymbol{H}) \propto$ $|F(\mathbf{H})|$ in the dynamical theory, where the integrated intensity is, in fact, proportional to the width of the Darwin curve in terms of $\omega$ while the Darwin curve width is proportional to $|F(\mathbf{H})|$. Note that when the zero-absorption condition is satisfied the proportionality $I(\boldsymbol{H}) \propto|F(\mathbf{H})|$ does not violate Eq. (1) because here both Eqs. (1) and (2) give the unity ratio [i.e., $I(\mathbf{H})=I(-\mathbf{H})]$. For absorbing crystals, the proportionality $I(\boldsymbol{H}) \propto|F(\mathbf{H})|$, in fact, is no longer valid. Since anomalous diffraction always involves significant $\mathrm{x}$-ray absorption, the intensity ratios of BPs always follow Eq. (1) instead of Eq. (2).

For a parallel-sided crystal plate with a finite thickness $t$, both wave fields exist in the crystal. Then the Bragg reflectivity is

$$
R(\mathbf{H}, \omega, \lambda, t)=|b|\left|\frac{r_{1}+r_{2} \Upsilon}{1+\Upsilon}\right|^{2}=\left|\frac{\Psi_{1}+\Psi_{2} \Upsilon}{1+\Upsilon}\right|^{2}\left|\frac{F(\mathbf{H})}{F(-\mathbf{H})}\right|,
$$

where $\Upsilon=-\left(\Psi_{1} / \Psi_{2}\right) \exp \left[i K\left(\delta_{1}-\delta_{2}\right) t\right]$ is the thickness function. Here, since the factor $\left|\left(\Psi_{1}+\Psi_{2} \Upsilon\right) /(1+\Upsilon)\right|^{2}$ is also symmetric about $\mathbf{H}$ and $-\mathbf{H}$, Eq. (9) again gives $R(\mathbf{H}, \omega, \lambda, t) / R(-\mathbf{H}, \omega, \lambda, t) \equiv|F(\mathbf{H})|^{2} /|F(-\mathbf{H})|^{2}$, which is equivalent to Eq. (8) for finite-thickness crystals.

Figure 4 shows the theoretical Darwin curves of the $\overline{2} 4 \overline{2} \overline{2} / 2 \overline{4} 22$ and $\overline{1} 2 \overline{1} \overline{1} / 1 \overline{2} 11$ pairs for a thin quartz ( $\overline{1} 2 \overline{1} \overline{1})$ wafer with $t=5 \mu \mathrm{m}$. Compared with Figs. 1(b) and 1(c),

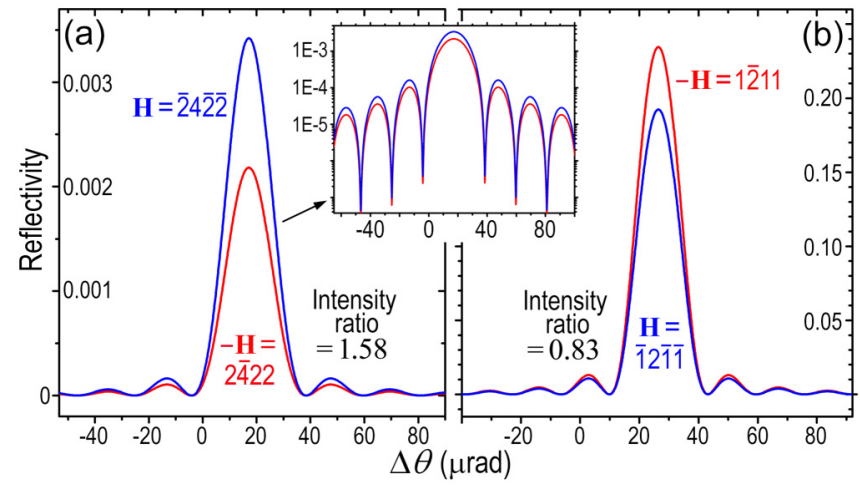

FIG. 4. Theoretical Darwin curves of a ( $(\overline{1} 2 \overline{1} \overline{1})$ quartz plate with thickness $t=5 \mu \mathrm{m}$. The diffraction conditions are the same as Figs. 1(b) and 1(c). (a) $\overline{2} 4 \overline{2} \overline{2} / 2 \overline{4} 22$ reflections. The inset shows the intensities on the logarithmic scale. (b) $\overline{1} 2 \overline{1} \overline{1} / 1 \overline{2} 11$ reflections.

here the Darwin curves are quite different, but the intensity ratios remain the same. In particular, the inset of Fig. 4(a) shows the interference fringes of the BP indeed have the same shape, as indicated by the exact coincidence of the positions of the intensity minima. Note that for the thickness of $5 \mu \mathrm{m}$ the diffraction process approaches the kinematical diffraction mechanisms. In fact, we have also used the kinematical diffraction mechanisms to simulate the Darwin curves for $t=5 \mu \mathrm{m}$, and the simulations are indeed close to the curves in Fig. 4. This proves that Eq. (1) is also valid for kinematical diffraction.

(a)
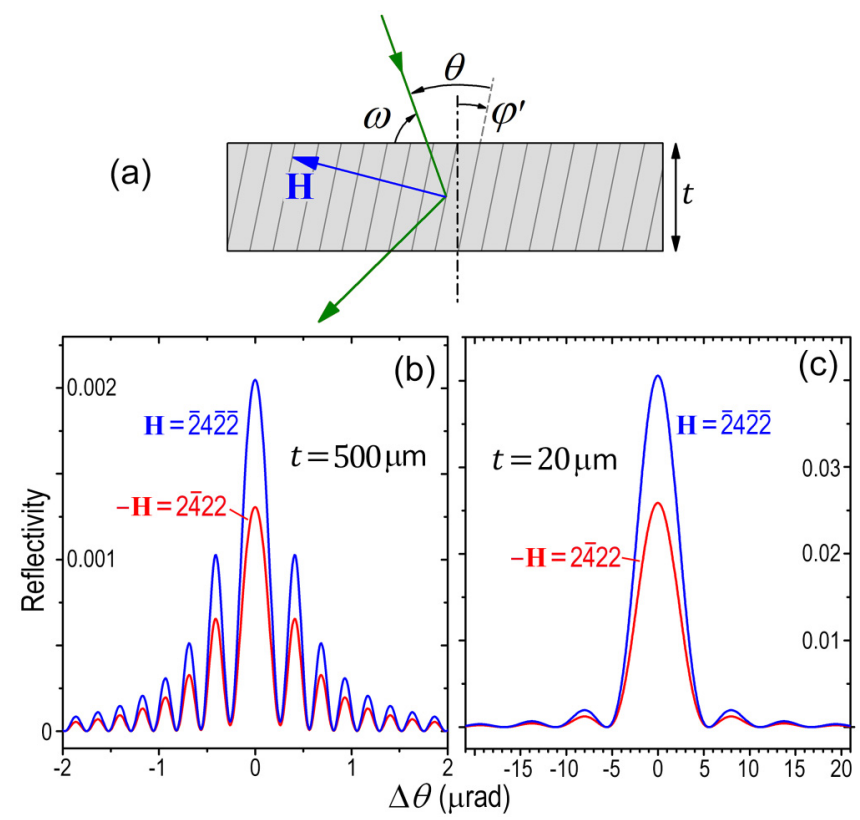

FIG. 5. (a) Schematic of Laue transmission diffraction. $\varphi^{\prime}$ is the asymmetric angle. (b) Theoretical diffraction curves of quartz $\overline{2} 4 \overline{2} \overline{2} / 2 \overline{4} 22$ for symmetric diffraction $\left(\varphi^{\prime}=0\right)$ in the thick-crystal case ( $t=500 \mu \mathrm{m}$, dynamical diffraction). (c) Thin-crystal case $(t=$ $20 \mu \mathrm{m}$, approaching kinematic diffraction). The intensity ratio is again 1.58 in both (b) and (c). 
For the Laue transmission diffraction geometry in Fig. 5(a), the normalized parameter is

$$
\begin{aligned}
\eta= & \frac{1}{C \Gamma[F(\mathbf{H}) F(-\mathbf{H}) b]^{1 / 2}}\left\{-\frac{\lambda}{d}\left[\cos \left(\omega-\varphi^{\prime}\right)-\frac{\lambda}{2 d}\right]\right. \\
& \left.-\frac{\Gamma F(\mathbf{0})(b-1)}{2}\right\},
\end{aligned}
$$

where $b=\cos \left(\theta_{B}+\varphi^{\prime}\right) / \cos \left(\theta_{B}-\varphi^{\prime}\right)$. Then the corrections to the two internal vertical wave vectors are

$$
\begin{aligned}
\delta_{1,2}= & \frac{C \Gamma[F(\mathbf{H}) F(-\mathbf{H})]^{1 / 2}}{2 \cos \left(\theta_{B}-\varphi^{\prime}\right) b^{1 / 2}}\left[\eta \pm\left(\eta^{2}+1\right)^{1 / 2}\right] \\
& +\frac{\Gamma F(\mathbf{0})}{2 \cos \left(\theta_{B}-\varphi^{\prime}\right)} .
\end{aligned}
$$

The reflectivity of reflection $\mathbf{H}$ is

$$
R_{\text {Laue }}(\mathbf{H}, t, \Delta \omega)=\left|\frac{F(\mathbf{H})}{F(-\mathbf{H})}\right| \frac{\left|\exp \left(i K \delta_{1} t\right)-\exp \left(i K \delta_{2} t\right)\right|^{2}}{4\left|\eta^{2}+1\right|},
$$

from which we again derive the same BP intensity ratio:

$$
\frac{R_{\text {Laue }}(\mathbf{H}, t, \Delta \omega)}{R_{\text {Laue }}(-\mathbf{H}, t, \Delta \omega)}=\frac{|F(\mathbf{H})|^{2}}{|F(-\mathbf{H})|^{2}} .
$$

Figures 5(b) and 5(c) show the calculated plane-wave Laue transmission diffraction rocking curves of the quartz $\overline{2} 4 \overline{2} \overline{2} / 2 \overline{4} 22$ BP. As expected, such diffraction curves have (angular) Pendellösung fringes besides the main peaks [17]. For both the thick-crystal case (dynamical diffraction) and the thin-crystal case (kinematic diffraction), the intensity ratio is again consistent with Eq. (1). In our paper, we have also used the Fourier coupled-wave diffraction theory (a more rigorous theory valid for any diffraction geometry, particularly grazing geometry) [23] to successfully verify Eq. (1) in various grazing-incidence diffraction configurations where the above formulas may fail.

In summary, we have demonstrated that the BP intensity ratios of noncentrosymmetric crystals are always equal to the squared modulus ratio of the two structure factors instead of their modulus ratio. In particular, the repeatable highresolution diffraction experiments of quartz crystals precisely proved the consistence between the theoretical and measured ratios. The dynamical theory reveals the underlying mechanism, in which the elementary diffraction function of the BP always obeys the $|F(\mathbf{H})|^{2} /|F(-\mathbf{H})|^{2}$ ratio rule. This is intrinsically valid for kinematical, dynamical, or mixed diffraction processes. Therefore, the BP intensity ratio is preserved for any practical convoluted or integrated intensities and for any diffraction geometry and incident beam conditions (even unfavorable). This indicates that under the same diffraction conditions the shapes of the two rocking curves of a BP must be identical, which is the most important criterion to ensure the reliability of the measurements (i.e., the rocking curves have not been affected by crystal defects or imperfections). The universal validity of Eq. (1) verified thus paves the way for various applications of $\mathrm{x}$-ray diffraction of BPs.

The experiments were carried out at beamline 1-BM of the Advanced Photon Source. We thank E. Kasman and M. Wojcik for help in preparation of the quartz samples and during the experiments. This work was supported by the US Department of Energy, Office of Science, Office of Basic Energy Sciences, under Contract No. DE-AC02-06CH11357. R.-W.P. was supported by the National Key R\&D Program of China (2017YFA0303702), and the National Natural Science Foundation of China (Grants No. 11634005 and No. 61975078).
[1] A. F. Peerdeman and J. M. Bijvoet, Acta Crystallogr. 9, 1012 (1956).

[2] G. L. Taylor, Acta Crystallogr. D 66, 325 (2010).

[3] M. Vijayan and S. Ramaseshan, International Tables for Crystallography (2010), Vol. B, Chap. 2.4, pp. 282-296.

[4] J. M. Bijvoet, A. F. Peerdeman, and A. J. van Bommel, Nature (London) 168, 271 (1951).

[5] S. L. Mair, P. R. Prager, and Z. Barnea, J. Appl. Crystallogr. 4, 169 (1971).

[6] M. Dudley, B. Raghothamachar, Y. Guo, X. R. Huang, H. Chung, D. T. J. Hurle, and D. F. Bliss, J. Cryst. Growth $\mathbf{1 9 2 ,}$ 1 (1998).

[7] R. W. W. Hooft, L. H. Stravera, and A. L. Spek, J. Appl. Crystallogr. 41, 96 (2008).

[8] Y. Tanaka, T. Takeuchi, S. W. Lovesey, K. S. Knight, A. Chainani, Y. Takata, M. Oura, Y. Senba, H. Ohashi, and S. Shin, Phys. Rev. Lett. 100, 145502 (2008).

[9] D. Creagh, Aust. J. Phys. 38, 371 (1985).
[10] T. Usui, Y. Tanaka, H. Nakajima, M. Taguchi, A. Chainani, M. Oura, S. Shin, N. Katayama, H. Sawa, Y. Wakabayashi, and T. Kimura, Nat. Mater. 13, 611 (2014).

[11] S. Gorfman, H. Simons, T. Iamsasri, S. Prasertpalichat, D. P. Cann, H. Choe, U. Pietsch, Y. Watier, and J. L. Jones, Sci. Rep. 6, 20829 (2016).

[12] X. R. Huang, S. S. Jiang, W. Zeng, X. B. Hu, W. J. Liu, D. Feng, H. C. Chen, Q. Z. Jiang, J. H. Jiang, and Z. G. Wang, Appl. Phys. Lett. 66, 2649 (1995).

[13] R. W. James, The Optical Principles of the Diffraction of X-Rays (Ox Bow, Woodbridge, 1982).

[14] W. H. Zachariasen, Theory of X-Ray Diffraction in Crystals (Wiley, New York, 1946).

[15] B. W. Batterman, Rev. Modern Phys. 36, 681 (1964).

[16] H. Cole and N. R. Stemple, J. Appl. Phys. 33, 2227 (1962).

[17] A. Authier, Dynamical Theory of X-Ray Diffraction (Oxford University, New York, 2001). 
[18] R. O. Freitas, C. F. Deneke, Â. Malachias, G. Darin, and S. L. Morelhão, J. Nanoparticle Res. 15, 1527 (2013).

[19] E. P. Warekois, M. C. Lavine, A. N. Mariano, and H. C. Gatos, J. Appl. Phys. 33, 690 (1962).

[20] X.-R. Huang, T. Gog, J. Kim, E. Kasman, A. H. Said, D. M. Casa, M. Wieczorek, M. G. Hönnicke, and L. Assoufid, J. Appl. Crystallogr. 51, 140 (2018).
[21] Y. Le Page and G. Donnay, Acta Crystallogr. B 32, 2456 (1976).

[22] Y. Shvyd'ko, X-Ray Optics: High-Energy-Resolution Applications, Springer Series in Optical Sciences (Springer-Verlag, Berlin, 2004).

[23] X.-R. Huang, R.-W. Peng, M. G. Hönnicke, and T. Gog, Phys. Rev. A 87, 063828 (2013). 